\title{
Adenosine Deaminase Two and Immunoglobulin M Accurately Differentiate Adult Sneddon's Syndrome of Unknown Cause
}

\author{
Gustavo C. Santo $^{a}$ Inês Baldeiras ${ }^{b, h}$ Rita Guerreiro ${ }^{c-e}$ Joana A. Ribeiro ${ }^{a}$ \\ Rosário Cunha $^{f}$ Taryn Youngstein $^{g}$ Sira Nanthapisalj, ${ }^{j}$ João Leitão ${ }^{h}$ \\ Carolina Fernandes $^{\mathrm{a}}$ Francisco Caramelo $^{\mathrm{i}}$ Maria do Rosário Almeida ${ }^{\mathrm{h}}$ \\ José Brás ${ }^{c-e}$ Isabel Santana ${ }^{a}$ b, h Centro Hospitalar e Universitário de Coimbra \\ ${ }^{a}$ Department of Neurology, Centro Hospitalar e Universitário de Coimbra, Coimbra, Portugal; ${ }^{b}$ Faculdade de \\ Medicina da Universidade de Coimbra, Coimbra, Portugal; ' UK Dementia Research Institute, University College \\ London, London, UK; ${ }^{d}$ Department of Molecular Neuroscience, UCL Institute of Neurology, University College \\ London, London, UK; ${ }^{e}$ Department of Medical Sciences and Institute of Biomedicine, iBiMED, University of Aveiro, \\ Aveiro, Portugal; ${ }^{f}$ Department of Clinical Pathology, Centro Hospitalar e Universitário de Coimbra, Coimbra, \\ Portugal; ${ }^{9}$ National Amyloidosis Centre, UCL School of Medicine, Royal Free Hospital, London, UK; ${ }^{\text {h } C e n t r o ~ d e ~}$ \\ Neurociências e Biologia Celular da Universidade de Coimbra, Coimbra, Portugal; 'Laboratório de Bioestatística e \\ Informática Médica da Faculdade de Medicina da Universidade de Coimbra, Coimbra, Portugal; jUCL Great Ormond \\ Street Institute of Child Health, London, UK; ${ }^{\mathrm{k}}$ Thammasat University, Bangkok, Thailand
}

\section{Keywords \\ Sneddon's syndrome - Cat eye syndrome chromosome region candidate 1 . Deficiency of adenosine deaminase 2 . Adenosine deaminase $2 \cdot$ Immunoglobulin M}

\begin{abstract}
Background: The association that exists between livedo reticularis (LR) and stroke is known as Sneddon's syndrome $(\mathrm{SnS})$. The disorder is classified as primary SnS (PSnS), if the cause remains unknown and secondary SnS. The condition is rare and it occurs mainly sporadically. In 2014, 2 independent teams described a new genetic disorder with childhood-onset, which was called deficiency of adenosine deaminase 2 (DADA2), characterized by recurrent fevers and vascular pathologic features that included LR and stroke. All the patients carried recessively inherited mutations in cat
\end{abstract}

\section{KARGER}

(c) 2019 S. Karger AG, Basel eye syndrome chromosome region candidate 1 gene (CECR1), encoding the adenosine deaminase 2 (ADA2) protein. Genetic testing is the standard for the diagnosis of DADA2. However, the diagnostic accuracy of more affordable laboratorial analysis in CECR1-mutated individuals remains to be established. We aim to determine whether plasma ADA2 activity and serum immunoglobulin M (lgM) levels can distinguish (1) DADA2 from other adult patients within the $\mathrm{SnS}$ spectrum, and (2) healthy CECR1 heterozygous ( $\mathrm{HHZ}$ ) from healthy controls $(\mathrm{HC})$. Methods: ADA2 activity in plasma and serum lgM concentrations was measured in adult patients within the SnS spectrum, healthy first-degree relatives and HC. Genetic results were used as the reference standard. The primary outcome measures were sensitivity and

G.C.S., I.B., and R.G. contributed equally to the manuscript. 
specificity derived from receiver operating curve analysis. Results: A total of 73 participants were included in the study: 26 patients with PSnS with no CECR1 mutation (PSnS), 6 biallelic (DADA2 patients) and $7 \mathrm{HHZ}$ CECR1 mutations and 34 $\mathrm{HC}$. Plasma ADA2 activity and serum IgM levels were significantly lower in DADA2 patients than in PSnS. With the use of the best indexes, plasma ADA2 activity differentiated PSnS from DADA2 with a sensitivity and specificity of $100.0 \%$ and $\mathrm{HHZ}$ from $\mathrm{HC}$ with a sensitivity of $97.1 \%$ and specificity of 85.7\%. Serum IgM levels also differentiated PSnS from DADA2 with a sensitivity of $85.2 \%$ and specificity of $83.3 \%$. Conclusion: Serum IgM levels might be used as a triage tool and plasma ADA2 activity performs perfectly as a diagnostic test for DADA2 in adult patients within the SnS spectrum. ADA2 activity in plasma also reliably distinguishes $\mathrm{HHZ}$ from $\mathrm{HC}$.

(c) 2019 S. Karger AG, Basel

\section{Introduction}

The association that exists between livedo reticularis (LR) and stroke is known as Sneddon's syndrome ( $\mathrm{SnS}$ ) [1].

The disorder is classified as primary $\mathrm{SnS}$ (PSnS) if the cause remains unknown, or secondary $\mathrm{SnS}$, which usually occurs as part of well-known autoimmune disorders (antiphospholipid syndrome with/without coexisting systemic lupus erythematous) [2].

$\mathrm{SnS}$ is rare and it occurs mainly sporadically [3]. In 2014, 2 independent teams $[4,5]$ described a new genetic disorder with childhood-onset, which was called Deficiency of adenosine deaminase (DADA2), characterized by recurrent fevers and vascular pathologic features that included LR and stroke. All the patients carried recessively inherited mutations in Cat Eye Syndrome Chromosome Region Candidate 1 gene (CECR1), encoding the adenosine deaminase 2 (ADA2) protein. Laboratory manifestations included reduced active ADA2 concentration in plasma and mild immunodeficiency. In the same year, Bras et al. [6] identified a compound heterozygous missense mutation in CECR1 in a Portuguese SnS family with adult onset, previously reported in 2002 [7, 8 ], expanding the phenotype associated with mutations in this gene to include later onsets of disease.

Genetic testing is the standard for the diagnosis of DADA2. However, the diagnostic accuracy of more affordable laboratorial analysis in CECR 1 mutated individuals remains to be established. The purpose of this study was therefore to determine whether plasma ADA2 activ- ity and serum immunoglobulin $\mathrm{M}$ (IgM) levels can distinguish DADA2 from other adult patients within the SnS spectrum and healthy CECR1 heterozygous (HHZ) from healthy controls ( $\mathrm{HC})$.

\section{Methods}

\section{Patient Selection}

All consecutive individuals within SnS's spectrum were collected in a prospective longitudinal database and full written informed consent was obtained from all participants. The study protocol was approved by the local Ethics Committee (Faculdade de Medicina da Universidade de Coimbra, Proc. CE-126/2015, approved November 30,2015$)$. The majority of participants were identified locally, in a tertiary University Hospital in Portugal. Three were referred by the National Amyloidosis Centre, UCL Division of Medicine, London.

Inclusion criteria were as follows: patients aged 18 years or older with SnS, both sporadic or familial; patients with LR and family history of SnS; healthy volunteers who were first-degree relatives of patients with familial SnS. We also included healthy volunteer individuals recruited by advertisement. Controls had no relationship with the other participants and no history of LR, cerebrovascular disease or other neurological illness.

$\mathrm{SnS}$ was defined by the association of a widespread LR involving the trunk and/or the buttock and/or the legs and at least one clinical cerebral event (either ischemic or haemorrhagic stroke, transient ischemic attack or a silent vascular brain lesion detected on MRI). Patients were excluded if they presented: (1) at least one of the clinical criteria and one of the laboratory criteria for Antiphospholipid antibody syndrome [9] and/or (2) clinical and immunological criteria for Systemic Lupus Erythematous [10] or (3) any thrombophilic state.

Demographic and clinical features, including vascular risk factors and laboratorial data, were collected from all participants in a standardized format.

All the technicians involved in the study were blinded for the clinical features and any other laboratorial results.

\section{Procedures}

Total ADA and ADA2 Activity in Plasma

Blood samples were collected from all participants into lithium heparin tubes. The tubes were centrifuged at $1,500 \mathrm{~g}$ at $4{ }^{\circ} \mathrm{C}$ for $10 \mathrm{~min}$ and plasma was separated and frozen at $-80^{\circ} \mathrm{C}$ until analysis.

Total ADA-T was determined through a commercial method (Diazyme) based on the conversion of adenosine into inosine and further into hypoxanthine (by purine nucleoside phosphorylase) and into uric acid and hydrogen peroxide (by xanthine oxidase). The conversion of hydrogen peroxide by peroxidase into the quinone dyes 4-aminoantipyrine and $\mathrm{N}$-ethyl-N-(2-hydroxy-3-sulfopropyl)$\mathrm{m}$-toluidine was then monitorized at $550 \mathrm{~nm}$ in a thermostatized spectrophotometer at $37^{\circ} \mathrm{C}$ (UVIKON $933 \mathrm{UV} /$ Visible). ADA2 activity was measured by the same method, in the presence of $0.1 \mathrm{mM}$ of erythro-9-(2-hydroxy-3-nonyl) adenine hydrochloride (EHNA, Sigma), a potent selective inhibitor of ADA1 [11]. ADA1 activity was then calculated by subtracting ADA2 activity from total ADA activity.
Santo et al. 
Each sample was run in duplicate and 2 control samples (with high and low levels of ADA activity) were assayed in each day for internal quality control. The intra-assay coefficient variation, calculated from 6 repeats, was $6.4 \%$, and the inter-assay coefficient variation, calculated for 13 runs performed, was $5.5 \%$.

\section{Quantitative Assessment of Serum IgM}

A nephelometric method was used for the measurement of IgM levels in serum samples, using the same technical conditions (performed on the same day and working session, and using the same batch of reagents, N ASIGM, Siemens HDP). The IgM contained in the human serum form immune complexes with specific antibodies that are present in the reagent. These complexes scatter a beam of light passed through the sample and the intensity of the scattered light is proportional to the concentration of IgM in the sample [12]. The results were then evaluated by comparison with a standard of known concentration.

\section{Genetic Analyses}

DNA was extracted according to manufacturer's instructions from saliva samples collected using the Oragene DNA (OG-500, DNA Genotek) kit or from blood samples using the GE Healthcare Nucleon $^{\mathrm{TM}}$ BACC3 Genomic DNA Extraction Kit. Exome and Sanger sequencing were used to identify mutations and variants in the CECR1 gene in all the patient's groups studied.

Exome sequencing was initially performed in 2 siblings from a Portuguese SS family and results were reported in Bras et al. [6] Exome sequencing was also performed in 14 Portuguese additional cases ( 4 familial and 10 sporadic) by preparing genomic DNA and capturing it using Agilent's SureSelect version 4 capture kit, according to manufacturer's instructions. Sequencing was performed in Illumina's HiSeq2000 using 100 bp paired-end reads and data were analyzed after quality control and following standard procedures (online suppl. data; for all online suppl. material, see www.karger.com/doi/10.1159/000495794). Variants in CECR1 were extracted and compared to databases of human genetic variability.

Sanger sequencing was used in the remaining samples. For this, primers for all exons of CECR1 were designed using Primer3. Details for the PCR conditions, electrophoresis of PCR products and Sanger sequencing procedures used and primer design are provided in the online supplementary material. Sanger sequencing of CECR 1 was also performed in the 3 patients that were referred by the National Amyloidosis Centre, UCL Division of Medicine, London. This was done at Great Ormond Street Hospital's Foundation Trust, using standard procedures.

\section{Statistical Analysis}

The results of genetic testing were the reference standard for the diagnosis of DADA2.

Baseline characteristics are presented as frequency (percentage) for dichotomous variables and mean \pm SD for continuous variables.

A 2-way Mann-Whitney U test was used to compare means between groups.

Area under the curve (AUC) for receiver operating characteristic (ROC) analysis was used to determine diagnostic accuracy (sensitivity, specificity, positive and negative predictive values, accuracy) for both tests (serum IgM levels and plasma ADA2 activ-

ADA2 and IgM Differentiate Adult SnS ity) with $95 \%$ CI. Cut-off points were set to minimize the difference between sensitivity and specificity (Youden index). Statistical significance was assumed at $p<0.05$.

\section{Results}

\section{Participants and Laboratorial Parameters}

Between January 2, 2016 and December 23, 2017, we screened 96 potential individuals; 73 of them (mean age $41.5 \pm 11.0$, male $31.5 \%$ ) met the inclusion criteria and were therefore enrolled in the study. Four subjects did not consent to participate and 19 were excluded as they had secondary SnS. Twenty-six patients with no CECR1 mutation (age $45.1 \pm 9.8$ ), 6 bi-allelic (DADA2 patients; age $41.7 \pm 12.1$ ) and 7 HHZ CECR1 mutated individuals (age $32.0 \pm 13.9$ ) and $34 \mathrm{HC}$ (age $40.6 \pm 10.2$ ) were included in the study (Fig. 1). The CECR1 mutations identified in the cohort studied and the coding variants identified in the individuals without CECR1 mutations are shown in online supplementary Tables S2, and S3 respectively.

General demographic and clinical characteristics of the study population are presented in Table 1.

Total ADA, ADA2 and ADA1 activities, as well as serum IgM levels of the studied population are shown in Table 2. DADA2 patients had significantly lower serum IgM levels $(p=0.002)$, as well as significantly reduced activity of total $\mathrm{ADA}(p<0.001)$ and ADA2 $(p<0.001)$ compared to PSnS patients. In fact, ADA2 activity was almost completely abolished in bi-allelic mutation carriers, ranging from 0 to 0.34 $\mathrm{U} / \mathrm{L}$ and showing no overlap with patients without mutation (range 5.15-25.41 U/L; Fig. 2b). For IgM, although a statistically significant difference was seen, still some overlap between groups remained (Fig. 2a). In HHZ, IgM levels were not significantly different from HCs $(p=0.225$; Fig. 2a); however, a significant reduction of both total ADA and ADA2 activity was seen $(p=0.004$ and $p<0.001$, respectively, vs. HC). The reduction in ADA2 activity in $\mathrm{HHZ}$ was not as strong as in DADA2 patients (activity levels ranging between 1.50 and $5.62 \mathrm{U} / \mathrm{L}$ ), and therefore some overlap with activitylevels determined in HCs was detected (Fig. 2b). No statistical differences were found in mean IgM levels or total ADA and ADA2 activity between PSnS and HC. ADA1 activity was similar between all groups.

\section{Diagnostic Accuracy of Plasma ADA2 Activity and Serum IgM Levels Differentiating DADA2 from PSnS}

An ROC curve was plotted to compare how effectively plasma ADA2 activity and serum IgM levels distinguished 
Fig. 1. Study profile. SnS, Sneddon's syndrome; PSnS, primary SnS; DADA2, deficiency of adenosine deaminase 2; HHZ, healthy CECR1 heterozygous; HC, healthy controls; CECR1, cat eye syndrome chromosome region candidate 1 gene.

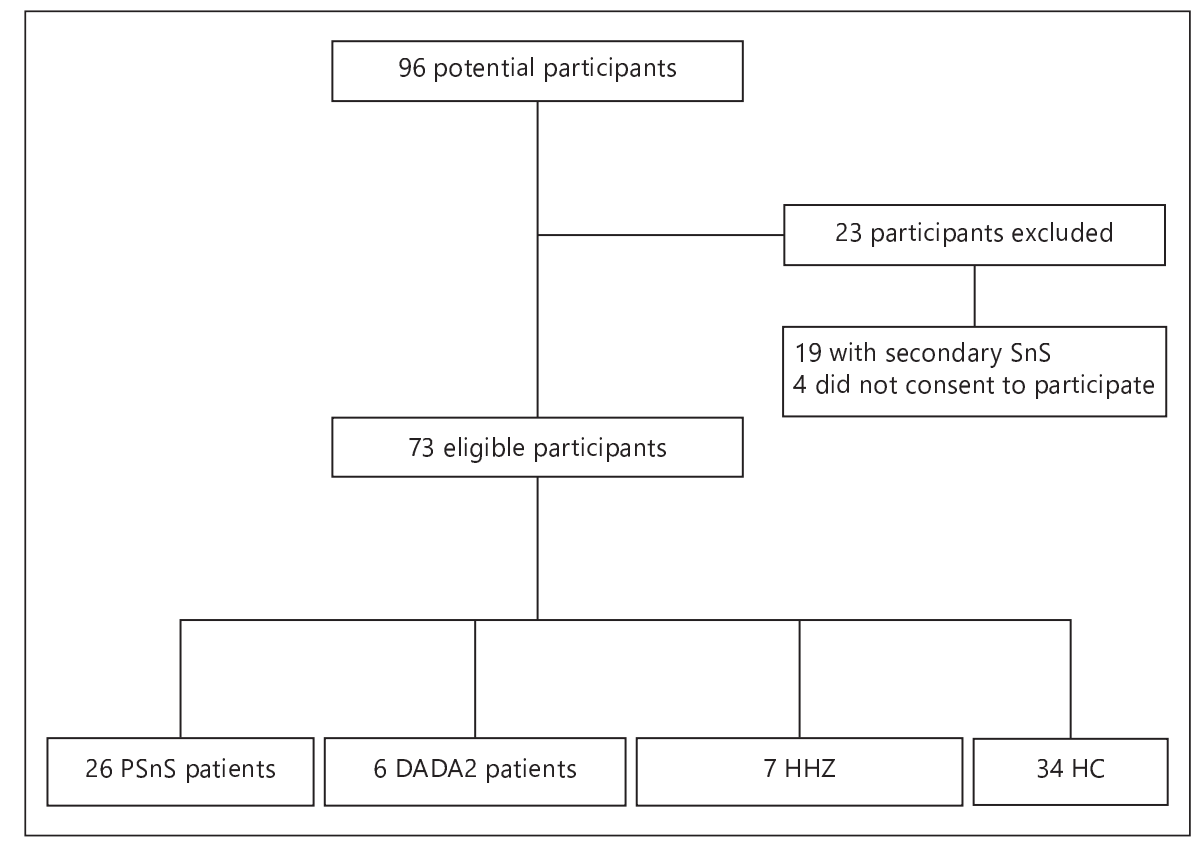

Table 1. Demographic and clinical characteristics of the cohort studied

\begin{tabular}{|c|c|c|c|c|c|}
\hline Variable & Total population & PSnS & DADA2 & $\mathrm{HHZ}$ & $\mathrm{HC}$ \\
\hline Patients, $n$ & 73 & 26 & 6 & 7 & 34 \\
\hline Age, years, mean \pm SD & $41.45 \pm 11.02$ & $45.12 \pm 9.78$ & $41.67 \pm 12.09$ & $32.00 \pm 13.87$ & $40.56 \pm 10.24$ \\
\hline Gender, male & $23(31.5)$ & $4(15.4)$ & $3(50.0)$ & $4(57.1)$ & $12(35.3)$ \\
\hline Hypertension & $21(28.8)$ & $11(42.3)$ & $3(50.0)$ & $0(0.0)$ & $7(20.6)$ \\
\hline Diabetes mellitus & $7(9.6)$ & $3(11.5)$ & $0(0.0)$ & $1(14.3)$ & $3(8.8)$ \\
\hline Dyslipidemia & $21(28.8)$ & $12(46.2)$ & $1(16.7)$ & $1(14.3)$ & $7(20.6)$ \\
\hline Smoking & $15(20.5)$ & $9(34.6)$ & $0(0.0)$ & $0(0.0)$ & $6(17.6)$ \\
\hline Alcoholism & $9(12.3)$ & $3(11.5)$ & $1(16.7)$ & $0(0.0)$ & $5(14.7)$ \\
\hline LR & $32(43.8)$ & $26(100.0)$ & $6(100.0)$ & $0(0.0)$ & $0(0.0)$ \\
\hline Raynaud phenomenon & $12(16.4)$ & $7(26.9)$ & $2(33.3)$ & $0(0.0)$ & $3(8.8)$ \\
\hline Migraine & $26(35.6)$ & $7(26.9)$ & $6(100.0)$ & $3(42.9)$ & $10(29.4)$ \\
\hline Recurrent fever & $7(9.6)$ & $2(7.7)$ & $5(83.3)$ & $0(0.0)$ & $0(0.0)$ \\
\hline Stroke & $29(39.7)$ & $26(100.0)$ & $4(66.7)$ & $0(0.0)$ & $0(0.0)$ \\
\hline
\end{tabular}

Data is represented as $n(\%)$, except when indicated otherwise.

PSnS, primary Sneddon's syndrome; DADA2, deficiency of adenosine deaminase 2; HHZ, healthy CECR1 heterozygous; HC, healthy controls; CECR1, cat eye syndrome chromosome region candidate 1 gene; LR, livedo reticularis/racemosa.

patients with DADA2 from PSnS (Fig. 3a). Accuracy parameters for this distinction are shown in Table 3. For serum IgM levels, at the best cut-off of $0.65 \mathrm{~g} / \mathrm{L}$, the AUC was 0.904 ( $95 \%$ CI $0.775-1.000)$, sensitivity of $85.2 \%$, and specificity of $83.3 \%(p=0.002)$. For plasma ADA2 activity, the best cut-off was fixed at $2.745 \mathrm{U} / \mathrm{L}$, with an AUC of 1.00 (95\% CI $1.000-1.000)$, sensitivity of $100.0 \%$ and specificity of $100.0 \%(p<0.001)$.
Differentiating HHZ from HC

To evaluate the diagnostic accuracy of these laboratorial parameters in differentiating $\mathrm{HHZ}$ from $\mathrm{HC}$, an ROC curve was also employed (Fig. 3b) and statistical parameters are shown in Table 3. For serum IgM levels, the ROC curve was not statistically different from the reference curve $(p=0.225)$. Conversely, for plasma ADA2 activity, ROC curve analysis fixed the best cut- 


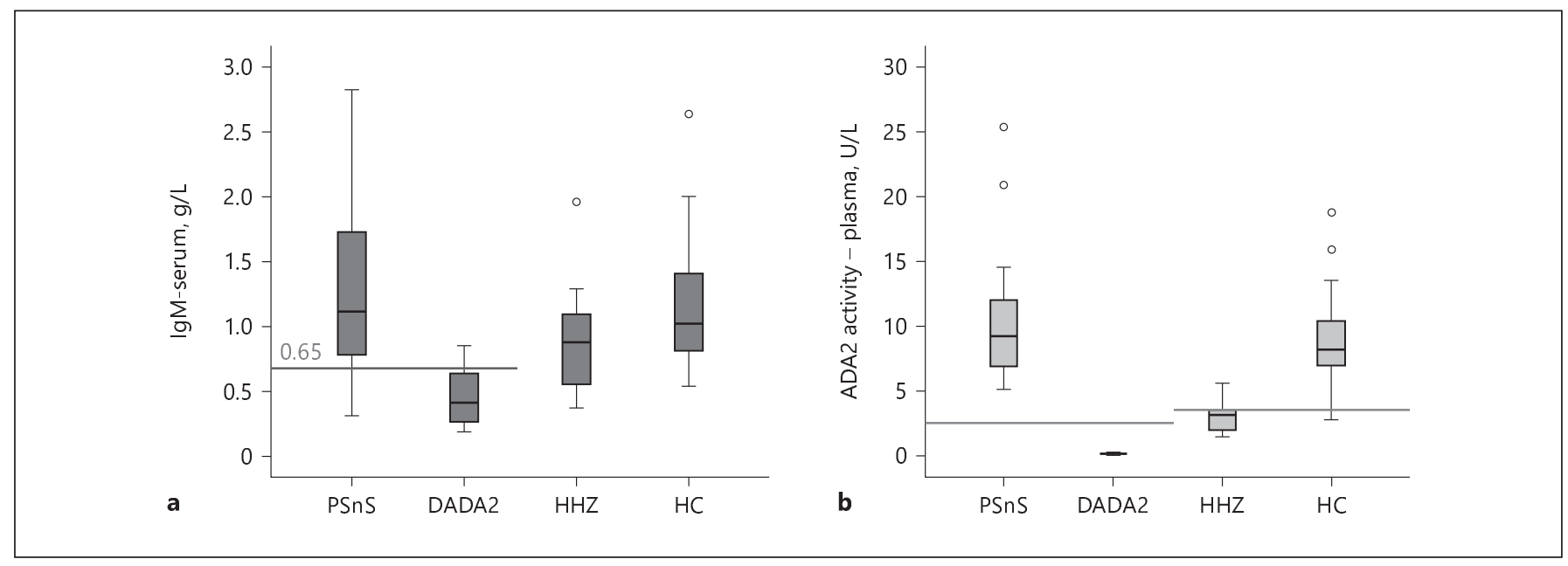

Fig. 2. a Serum IgM concentration in patients with PSnS and DADA2 patients, HHZ and HC. Box plots show 10th, 25th, 50th, 75th and 90th percentiles; the dots represent outliers. Gray line, cut-off level for differentiating PSnS from DADA2 patients; (b) Plasma ADA2 activity in patients with $\mathrm{PSnS}$ and DADA2 patients, HHZ and HC. Box plots show 10th, 25th, 50th, 75th and 90th per- centiles; the dots represent outliers. Gray line, cut-off level for differentiating PSnS from DADA2 patients and HHZ from HC; PSnS, primary SnS; DADA2, deficiency of adenosine deaminase 2; HHZ, healthy CECR1 heterozygous; HC, healthy controls; CECR1, cat eye syndrome chromosome region candidate 1 gene; ADA2, adenosine deaminase 2; IgM, immunoglobulin $\mathrm{M}$.

Table 2. ADA-T, ADA2 and ADA1 activity in plasma and serum IgM concentration of the studied population

\begin{tabular}{lccccrr}
\hline & PSnS & DADA2 & $\begin{array}{l}p \text { value, } \\
\text { PSnS vs. DADA2 }\end{array}$ & HHZ & HC & $\begin{array}{c}p \text { value, } \\
\text { HHZ vs. HC }\end{array}$ \\
\hline Patients, $n$ & 26 & 6 & - & 7 & 34 & - \\
ADA-T, U/L & $14.5 \pm 5.4$ & $4.0 \pm 1.6$ & $<0.001$ & $7.8 \pm 2.9$ & $12.5 \pm 3.3$ & 0.004 \\
ADA2, U/L & $10.3 \pm 4.6$ & $0.1 \pm 0.2$ & $<0.001$ & $3.0 \pm 1.4$ & $8.9 \pm 3.3$ & $<0.001$ \\
ADA1, U/L & $4.2 \pm 1.2$ & $3.9 \pm 1.6$ & 0.847 & $4.8 \pm 1.9$ & $3.6 \pm 1.3$ & 0.089 \\
IgM, g/L & $1.3 \pm 0.7$ & $0.5 \pm 0.3$ & 0.003 & $0.9 \pm 0.5$ & $1.1 \pm 0.5$ & 0.225 \\
\hline
\end{tabular}

Data is shown as mean $\pm \mathrm{SD}$.

ADA-T, total ADA activity in plasma; ADA2, ADA2 activity in plasma; ADA1, ADA1 activity in plasma; ADA, adenosine deaminase; PSnS, primary Sneddon's syndrome; DADA2, deficiency of adenosine deaminase 2; HHZ, healthy CECR1 heterozygous; HC, healthy controls; CECR1, cat eye syndrome chromosome region candidate 1 gene; ADA1, adenosine deaminase 1; ADA2, adenosine deaminase 2; IgM, immunoglobulin $\mathrm{M}$.

off at $3.77 \mathrm{U} / \mathrm{L}$, with an AUC of 0.971 (95\% CI $0.923-$ $1.000)$, sensitivity of $97.1 \%$ and specificity of $85.7 \%(p<$ $0.001)$.

\section{Discussion}

In the present study, we found that in adult SnS patients, plasma ADA2 activity was significantly lower in DADA2 in comparison with PSnS patients. An extremely low, or even absent, activity of ADA2 in paediatric patients with DADA2

ADA2 and IgM Differentiate Adult SnS had previously been shown in other studies [13-15]. These authors have previously shown a statistically significant difference between ADA2 activity in DADA2 patients in comparison to $\mathrm{HC}$, but the relation with sporadic patients exhibiting a similar clinical phenotype was not tested. In fact, the diagnostic accuracy of ADA2 activity in differentiating DADA2 from sporadic PSnS in adults had never been investigated. In our study, we show that, at the best cut-off of $2.745 \mathrm{U} / \mathrm{L}$, plasma ADA2 activity distinguished DADA2 patients from PSnS with $100 \%$ sensitivity and $100 \%$ specificity, which suggests that it fits perfectly as a diagnostic test.

Cerebrovasc Dis 2018;46:257-264 

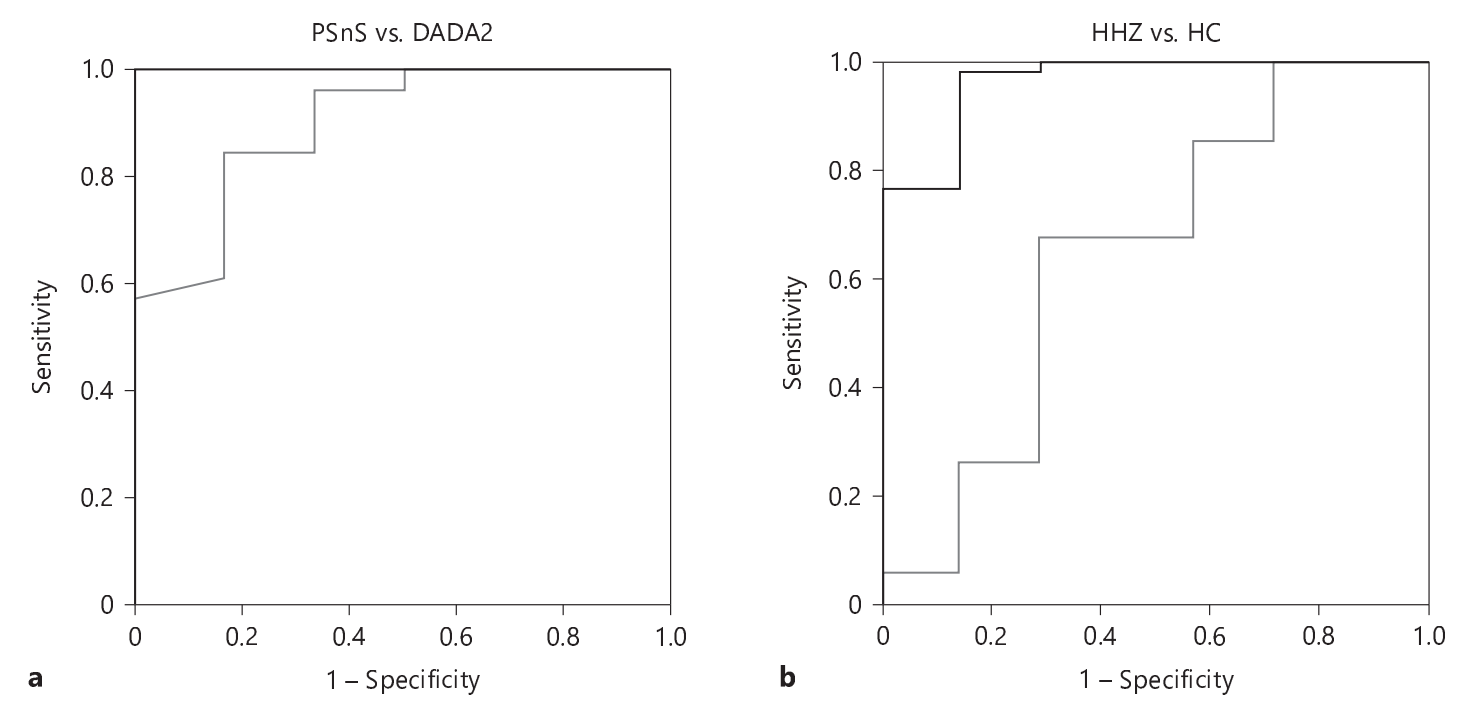

Fig. 3. ROC curve for laboratorial parameters. a PSnS vs. DADA2 patients: plasma ADA2 activity (black line) and serum IgM concentration (grey line); (b) HHZ vs. HC: plasma ADA2 activity (black

line) and serum IgM concentration (grey line). PSnS, primary Sneddon's syndrome; DADA2, deficiency of adenosine deaminase 2; HHZ, healthy CECR1 heterozygous; HC, healthy controls.

Table 3. AUC, sensitivity, specificity, and positive and negative predictive values for receiver operating characteristic curves using best laboratorial tests

\begin{tabular}{lcc}
\hline & PSnS vs. DADA2 & HHZ vs. HC \\
\hline ADA2 activity in plasma, U/L & & \\
AUC (95\% CI) & $1.00(1.00-1.00)$ & $0.971(0.923-1.00)$ \\
Sensitivity, \% & 100 & 97.1 \\
Specificity, \% & 100 & 85.7 \\
PPV, \% (95\% CI) & $100(100.0-100.0)$ & $71.4(38.1-91.0)$ \\
NPV, \% (95\% CI) & $100(100.0-100.0)$ & $97.7(87.8-99.6)$ \\
Accuracy, \% (95\% CI) & 100 & $94.1(83.8-98.8)$ \\
Serum IgM concentration, g/L & & $0.647(0.386-0.908)$ \\
AUC (95\% CI) & $0.901(0.767-1.000)$ & 67.6 \\
Sensitivity, \% & 85.2 & 71.4 \\
Specificity, \% & 83.3 & $67.4(35.5-91.9)$ \\
PPV, \% (95\% CI) & $83.3(40.2-97.4)$ & $68.3(59.6-74.8)$ \\
NPV, \% (95\% CI) & $85.2(73.4-92.3)$ & $86.7)$ \\
Accuracy, \% (95\% CI) & $84.8(68.1-94.9)$ & \\
\hline
\end{tabular}

PSnS, primary Sneddon's syndrome; DADA2, deficiency of adenosine deaminase 2; HHZ, healthy CECR1 heterozygous; HC, healthy controls; CECR1, cat eye syndrome chromosome region 1 gene; AUC, area under the curve; ADA2, adenosine deaminase 2; PPV, positive predictive value; NPV, negative predictive value; IgM, immunoglobulin M.

Although the pathogenesis of DADA2 is still unclear, there is some evidence that the underlying mechanism involves the loss-of-function mutations in CECR1 encoding the ADA2 protein [5]. ADA2 is known to be produced by myeloid cells and is a growth factor for endothelial and leukocyte development [4]. In fact, despite CECR1 not being expressed in the endothelium, DADA2 leads to a defect in endothelial instability and integrity [16] in the small and medium vessels of patients with ADA2 mutations as well as impairment of M2 macrophage differen- 
tiation [4]. Once secreted, it induces the differentiation of monocytes into macrophages, possibly by binding proteoglycan-like structures on the cellular surface [17]. Moreover, ADA2 seems to be involved in the balance between pro-inflammatory (M1) and anti-inflammatory (M2) monocytes. Its absence has been associated with a defect in differentiation of M2 macrophages, which leads to a prevalence of pro-inflammatory M1 cells [18], establishing a vicious circle of vasculopathy and inflammation [4].

As previously stated, a mild immunodeficiency can also be observed in DADA2, and indeed, very recently, Schepp et al. [19] suggested that patients with antibody deficiency should be screened for DADA2. Some patients present hypogammaglobulinemia that may affect IgM or all Ig subclasses [18]. In our study, for serum IgM levels, at the best cut-off of $0.65 \mathrm{~g} / \mathrm{L}$, the AUC to differentiate between DADA2 and sporadic PSnS patients was 0.904 with a sensitivity of $85.2 \%$, and specificity of $83.3 \%$, which suggests that it has an excellent diagnostic accuracy.

These results are particularly relevant, since recent observational studies have reported that some drugs (antiTNF- $\alpha$ with or without daily prednisolone and/or an alternative disease modifying anti-rheumatic drug) [14] seem to be effective, and hematopoietic stem cell transplantation might represent a potential definitive treatment for DADA2 [16].

It should also be mentioned that, although the clinical phenotype of monoallelic CECR1 mutated subjects is not clear, some authors report a full picture of a DADA2-like disease in some of those individuals [18]. This was not the case for individuals found in this study as heterozygous carriers of CECR1 mutations. It is possible though that these subjects will come to develop some features of the disease as they age.

In the present study, for plasma ADA2 activity, at the best cut-off of $3.77 \mathrm{U} / \mathrm{L}$, the AUC for differentiating between $\mathrm{HHZ}$ and $\mathrm{HC}$ was 0.971 , with a sensitivity of $97.1 \%$ and a specificity of $85.7 \%$, which corresponds to an almost perfect test. Other studies have reported on ADA2 activity in CECR1 single-mutation carriers, and while some authors showed a reduction to approximately half $[4,19]$ other reported no difference in relation to HCs [5, 14]. Meanwhile, for serum IgM levels, at the best cut-off of $0.92 \mathrm{~g} / \mathrm{L}$, AUC was 0.647 ( $p=0.225,95 \%$ CI $0.386-$ 0.908 ), with a sensitivity of $67.6 \%$, and specificity of 71.4 $\%$, which suggests that this test does not accurately identify CECR 1 heterozygous.

Our study has some important strengths. First, it was prospectively designed and the procedures of screening and confirmation were standardized according to the protocol. Second, the plasma ADA2 activity and the serum IgM measurements were determined in all subjects and each of the tests was performed by the same raters, using the same method and under equal technical conditions. Third and the most relevant, we tried to establish, for the first time, the accuracy of these laboratorial tests to distinguish between adult DADA2 and PSnS but also to differentiate $\mathrm{HHZ}$ individuals from HCs, together with the optimal cut-off points. Notwithstanding, these findings should be confirmed in larger multicentre studies, that would encompass both adult and paediatric DADA2 patients. As other authors have reported, ADA2 activity might be lower in adults that in children $[4,14]$ and an age-adjusted cut-off value would probably be needed. In our study, as in the study of Schepp et al. [19], there was no evidence of an influence of the position and type of mutation on ADA2 enzymatic activity. However, this should also be confirmed by the inclusion of patients with other CECR1 mutations.

This study also has some limitations. As it is presumably a rare disorder, the number of patients with bi-allelic and heterozygous mutations was low, which might have biased the data interpretation. For the same reason, we did not perform genetic testing in healthy volunteers.

In conclusion, serum IgM measurement has a potential role as a primary screening tool and plasma ADA2 activity might be an excellent diagnostic test for DADA2 in patients with $\mathrm{SnS}$ of unknown cause, making them suitable for implementation in clinical practice. ADA2 activity in plasma also provides high accuracy to identify CECR1 heterozygous individuals.

\section{Acknowledgements}

The authors would like to thank Graça Fernandes from the nurse staff and Rui Pascoal for the technical assistance.

\section{Disclosure Statement}

The authors have no conflicts of interest to declare relevant to the manuscript.

\section{Funding Source}

R.G. and J.B. work is funded by Research Fellowships from the Alzheimer's Society and J.L. by the Portuguese Foundation for Science and Technology (ref. PD/BD/13108/2017). 


\section{Authors Contribution}

G.C.S., I.B., and J.A.R.: designed the study. G.C.S., I.B., C.F., and R.G.: wrote the article. F.C. and G.C.S.: performed the statistical analysis. J.B., R.G., S.N., and M.R.A.: performed and analysed the genetic data. I.B. and J.L.: performed and analysed the plasma ADA2 activity. R.C.: performed and analysed the serum IgM concentration. G.C.S., J.A.R., and T.Y.: performed the clinical assessments. I.S.: reviewed the manuscript for content. All authors read the manuscript, reviewed and made significant contributions.

\section{References}

1 Sneddon IB: Cerebro-vascular lesions and livedo reticularis. Br J Dermatol 1965;77:180-185.

2 Schellong SM, Weissenborn K, Niedermeyer J, Wollenhaupt J, Sosada M, Ehrenheim C, et al: Classification of Sneddon's syndrome. Vasa 1997;26:215-221.

3 Wu S, Xu Z, Liang H: Sneddon's syndrome: a comprehensive review of the literature. Orphanet J Rare Dis 2014;9:1-7.

4 Zhou Q, Yang D, Ombrello AK, Zavialov AV, Toro C, Zavialov AV, et al: Early-onset stroke and vasculopathy associated with mutations in ADA2. N Engl J Med 2014;370:911-920.

5 Navon Elkan P, Pierce SB, Segel R, Walsh T, Barash J, Padeh S, et al: Mutant adenosine deaminase 2 in a polyarteritis nodosa vasculopathy. N Engl J Med 2014;370:921-931.

6 Bras J, Guerreiro R, Santo G: Mutant ADA2 in vasculopathies. N Engl J Med 2014;371: 478-480.

7 Santo GC, Mascarenhas R, Geraldo A, et al: Famillial Sneddon's syndrome: clinical, dermatologic and radiographic findings. Cerebrovasc Dis 2002;13(suppl 3):50.

8 Mascarenhas R, Santo G, Gonçalo M, Ferro MA, Tellechea O, Figueiredo A: Familial Sneddon's syndrome. Eur J Dermatol 2003; 13:283-287.
9 Keeling D, Mackie I, Moore GW, Greer IA, Greaves M; British Committee for Standards in Haematology: Guidelines on the investigation and management of antiphospholipid syndrome. Br J Haematol 2012;157:47-58.

10 Kuhn A, Bonsmann G, Anders HJ, Herzer P, Tenbrock K, Schneider M: The Diagnosis and Treatment of Systemic Lupus Erythematosus. Dtsch Ärztebl Int 2015;112:423-432.

11 Muraoka T, Katsuramaki T, Shiraishi H, Yokoyama MM: Automated enzymatic measurement of adenosine deaminase isoenzyme activities in serum. Anal Biochem 1990;187: 268-272.

12 Mali B, Armbruster D, Serediak E, Ottenbreit T: Comparison of immunoturbidimetric and immunonephelometric assays for specific proteins. Clin Biochem 2009;42:15681571.

13 Van Eyck L, Hershfield MS, Pombal D, Kelly SJ, Ganson NJ, Moens L, et al: Hematopoietic stem cell transplantation rescues the immunologic phenotype and prevents vasculopathy in patients with adenosine deaminase 2 deficiency. J Allergy Clin Immunol 2015;135; 283-287.

14 Nanthapisal S, Murphy C, Omoyinmi E, Hong Y, Standing A, Berg S, et al: Deficiency of adenosine deaminase type 2: a description of phenotype and genotype in fifteen cases. Arthritis Rheumatol 2016;68:2314-2322.

15 Hashem H, Egler R, Dalal J: Refractory pure red cell aplasia manifesting as deficiency of adenosine deaminase 2. J Pediatr Hematol Oncol 2017;39:e293-e296.

16 Hashem H, Kumar AR, Müller I, Babor F, Bredius R, Dalal J, et al: Hematopoietic stem cell transplantation rescues the hematological, immunological, and vascular phenotype in DADA2. Blood 2017;130: 2682 2688.

17 Oda H, Kastner DL: Genomics, biology, and human illness: advances in the monogenic autoinflammatory diseases. Rheum Dis Clin North Am 2017;43:327-345.

18 Caorsi R, Penco F, Grossi A, Insalaco A, Omenetti A, Alessio M, et al: ADA2 deficiency (DADA2) as an unrecognised cause of early onset polyarteritis nodosa and stroke: a multicentre national study. Ann Rheum Dis 2017;76:1648-1656.

19 Schepp J, Bulashevska A, Mannhardt-laakmann W, Cao H, Yang F, Seidl M, et al: Deficiency of adenosine deaminase 2 causes Antibody Deficiency. J Clin Immunol 2016;36: 179-186. 